\title{
Anti-inflammatory effect of methanol extracts of hemp leaf in IL-1 $\beta$-induced synovitis
}

\author{
Natthachai Duangnin ${ }^{1,2}$, Jeerawan Klangjorhor ${ }^{1}$, Prapatsorn Tipparat ${ }^{2}$, Sarita \\ Pinmanee $^{3}$, Thanyaluck Phitak ${ }^{1}$, Peraphan Pothacharoen ${ }^{1}$ and Prachya \\ Kongtawelert ${ }^{1 *}$ \\ ${ }^{1}$ Thailand Excellence Center for Tissue Engineering and Stem Cells, Chiang Mai University, ${ }^{2}$ The 1st Regional Medical \\ Sciences Center Chiang Mai, ${ }^{3}$ Highland Research and Development Institute (Public Organization), Chiang Mai, Thailand
}

*For correspondence: Email: prachya.kongtawelert@gmail.com; Tel: +66-53-894-188

Revised accepted: 16 June 2017

\begin{abstract}
Purpose: To examine the effectiveness of some hemp (Canabis sativa) leaf extracts as an antiinflammatory agent on synovitis in vitro.

Methods: Synovial fibroblast cell line SW982 was induced with $5 \mathrm{ng} / \mathrm{mL}$ of interleukin 1-beta (IL-1ß) to trigger cellular inflammation. The cells were then treated with prepared extracts of hemp (Canabis sativa) leaf originating from three different cultivation sites with varying proportions of terpenoids and cannabinoids, especially cannabidiol (CBD) and tetrahydrocannabinol (THC). Nitric oxide (NO) and prostaglandin E2 (PGE2) production as well as expression of inducible nitric oxide synthase (iNOS), cyclooxygenase-2 (COX-2), and IL-1 $\beta$ genes were determined.

Results: All hemp extracts reduced the production of NO and PGE2 in a dose-dependent manner. The expressions of iNOS, COX-2 and IL-1 3 genes were significantly decreased by the hemp extracts. This effect was likely related to the amount of sesquiterpenoids and THC. The extract from Huai Hoi (HH) cultivar showed the most promising results for further studies.

Conclusion: The extracts of hemp leaf substantially reduces the level of biomarkers for inflammation in vitro. Therefore, the extracts have a potential application as an inflammatory counteractant in synovitis.
\end{abstract}

Keywords: Canabis sativa, Hemp, Synovitis, Cannabinoids, Nitric oxide, Interleukin-1 beta

\begin{abstract}
Tropical Journal of Pharmaceutical Research is indexed by Science Citation Index (SciSearch), Scopus, International Pharmaceutical Abstract, Chemical Abstracts, Embase, Index Copernicus, EBSCO, African Index Medicus, JournalSeek, Journal Citation Reports/Science Edition, Directory of Open Access Journals (DOAJ), African Journal Online, Bioline International, Open-J-Gate and Pharmacy Abstracts
\end{abstract}

\section{INTRODUCTION}

Osteoarthritis $(O A)$ is a well-known inflammationrelated disease. It is a chronic disease associated with joint degeneration and reparative bone response on chondrocytes, subchondral bones, and synoviocytes [1]. The inflammation of synoviocytes, or synovitis, is known to be related to $\mathrm{OA}$ and can be used as a diagnostic marker [2]. In terms of physiology, synoviocytes manage to protect and maintain the integrity of articular cartilage surfaces, which are altered in OA [3]. In addition, synoviocytes in OA becomes a source of proinflammatory cytokines including interleukin 1-beta (IL-1 $\beta$ ) and nitric oxide (NO). There is strong evidence indicating that $\mathrm{IL}-1 \beta$ alone, and probably together with tumor necrosis factor alpha (TNF- $\alpha$ ), triggers joint destructive mechanism as both cytokines are dominantly expressed in OA synoviocytes, synovial fluid and cartilage [4].

The production of $\mathrm{NO}$ and the expression of inducible nitric oxide synthase (iNOS) gene have also been found to escalate in many inflammatory disorders including OA [5]. 
Moreover, NO can stimulate the activity of the cyclooxygenase-2 (COX-2) enzyme and enhance production of inflammatory mediators including prostaglandin $\mathrm{E}_{2}$ (PGE2) [6]. Current $\mathrm{OA}$ therapeutic guidelines focus on regulating the activity of related enzymes that produce proinflammatory cytokines. The commonly used drugs are glucocorticoids, including dexamethasone, and COX-2 inhibitors, but these therapeutic agents produce undesired side effects [7].

Hemp (Canabis sativa L.) is a versatile plant used for industrial, agricultural and medicinal purposes since ancient times [8]. In pharmacology, hemp has been applied to numerous diseases, especially pain and neuronal disorders $[9,10]$. The active compounds in hemp plant are known as cannabinoids and the most potent one is tetrahydrocannabinol (THC). THC affects the neurons which triggers delusion and delirium, known as the "psychotomimetic effect". Another important substance in hemp is non-psychoactive cannabinoid known as cannabidiol (CBD). THC and $\mathrm{CBD}$ together can reduce the progression and severity of pain in various diseases when used in appropriate proportions, e.g., when used in a ratio of $1: 1$ or $2: 1$ [11]. The action of the cannabinoids occurs through their specific receptors, cannabinoid receptors 1 and 2 (CB1 and CB2), which are normally expressed in brain, neuron cells and immune cells [12]. The activation of these receptors affect the secretion of cytokines from cells but the exact role of $\mathrm{CB}$ receptors, especially CB2, is still unclear [13]. Interestingly, CB2 has been recently reported to be involved in the occurrence and severity of inflammation, including arthritis [14]. Therefore, cannabinoids and other compounds contained in hemp might be effective on synovitis.

Although many parts of the hemp plant possess cannabinoids, most of the researchers and manufacturers are interested in hemp oil or hemp seeds [15]. Hemp leaves are usually disposed in both industrial and pharmaceutical processes. In this study, we examined the phytochemical content, especially THC and CBD, in the leaves of hemp cultivated in Thailand. The phytochemicals in hemp leaf extracts were discovered using gas chromatography and mass spectrophotometry (GC/MS) and the extracts were investigated for their anti-inflammatory effect in synovitis induced by IL-1 $1 \beta$.

\section{EXPERIMENTAL}

This study was approved by the Narcotics Control Division, Food and Drug Administration,
Ministry of Public Health, Thailand (approval ref.no. 2100626014000000). All activities undertaken complied with the restrictions of the National Narcotics and Psychotropic Substances Act. The participating institutes have been authorized to import, cultivate, and possess hemp for research and educational purposes under license nos. THAI.FDA.A4.0589033, THAI.FDA.A4.0589026, and THAI.FDA.A4.05890 25 , respectively.

\section{Hemp cultivation and extraction}

The hemp seeds in this study were collected from three areas of Chiang Mai Province, which differed from each other in geography and agronomy. The seed varieties were identified by their origins: (1) Huai Hoi $(\mathrm{HH})$ (N18.623974 E98.655991), (2) Mae Sa Mai (MSM) (N18.885391 E98.84600), and (3) Pang Ung (PU) (N18.783784 E98.159269). All hemp seeds were further cultivated in the Pangda Royal Agriculture Station in Samoeng, Chiang Mai (N18.854925 E98.760765). After 90 days of cultivation (June to September), the leaves on the upper third of the main stem were collected. All plant cultivars were sampled and identified by a botanist at the Herb Museum, Faculty of Pharmacy, Chiang Mai University (voucher no. 023209). The leaves were dried at $40 \stackrel{\circ}{\circ}$ for $48 \mathrm{~h}$ and were ground and sieved through 32-mesh sieving. For extraction, $100 \mathrm{~g}$ of dried leaf powder was percolated with $400 \mathrm{ml}$ methanol for $24 \mathrm{~h}$. The extracts were collected and filtered through Whatman's filter paper No.1. The extraction process was repeated until the percolated solvent was clear. Methanol was then eliminated using a vacuum evaporator and the yield of collected extracts was measured. All compounds in the hemp extracts were identified using NIST library provided with the GC/MS apparatus [16].

\section{Human synovial fibroblast cell line (SW982) culture and treatment}

SW982 was acquired from ATCC ${ }^{\circledR}$ number HTB93 and was authenticated by DiagCor Bioscience Incorporation Limited. The cells were retained in Leibovitz's L-15 culture media complemented with $10 \%$ fetal bovine serum, $100 \mathrm{U} / \mathrm{ml}$ penicillin $\mathrm{G}$ sodium and $100 \mu \mathrm{g} / \mathrm{mL}$ streptomycin. The cells were kept in a $\mathrm{CO}_{2}$ free incubator at $37^{\circ} \mathrm{C}$. Prior to the experiments, the cells were plated in 6-well plates at a concentration of $2 \times 10^{5}$ cells per well. Phytochemicals at appropriate concentrations were used to treat the cells either with or without $5 \mathrm{ng} / \mathrm{mL}$ of IL-1 $1 \beta$, which was added for $24 \mathrm{~h}$ to induce synovitis. Dexamethasone at a concentration $100 \mathrm{nM}$ was used as the positive 
control. After the treatment period, cell lysates were collected for determination of gene expression while culture supernatants were collected for measurement of released cytokine.

\section{Nitric oxide determination}

Prior to the experiment, appropriate doses of the hemp extracts were determined using MTT assay [17]. The total amount of released nitric oxide was measured via nitrite which accumulated in the culture supernatant. After the cell treatment, $1 \mathrm{~mL}$ of culture supernatant was immediately collected and Griess reagent $(0.1 \%$ $\mathrm{N}$-(1-naphthyl) ethylenediamine dihydrochloride, $1 \%$ sulfanilamide, and $2.5 \% \mathrm{H}_{3} \mathrm{PO}_{4}$ ) was added in an equal volume. The mixture was stored for 10 minutes at ambient temperature and then measured for absorbance at $540 \mathrm{~nm}$. The nitrite concentration in the samples was calculated using the standard curve generated from sodium nitrite concentrations ranging from 1 to $100 \mu \mathrm{M}$.

\section{Prostaglandin E2 determination}

The level of produced PGE2 was measured using a PGE2 competitive ELISA kit purchased from Thermo Fisher Scientific Co., Ltd., Thailand. The culture media acquired from the treated cells were collected and transferred onto the analytical plate provided with the kit. The experimental procedure was performed following the manufacturer's guidelines. Absorbance was measured at UV $405 \mathrm{~nm}$. The level of PGE2 concentration in the samples was interpreted from the standard curve, which was generated from the standard PGE2 at a concentration range of 0 to $5000 \mathrm{pg} / \mathrm{mL}$.

\section{Gene expression analysis}

After treating the cells, total RNA was separated using the Illustra RNAspin Mini RNA Isolation Kit. Reverse transcription reaction was operated using $1.0 \mu \mathrm{g}$ of total RNA and then reversetranscribed into cDNA using the Tetro cDNA synthesis kit. Real-time reverse transcriptase polymerase chain reaction technique (RT-PCR) was conducted using SensiFAST ${ }^{T M}$ SYBR $^{\circledR}$ LoROX kit. The reactions were conducted to determine the process of denaturation, annealing and extension on real-time RT-PCR detection system using 7500 Fast Real-Time PCR instrument. The data were collected and the fold changes were measured via the $2^{-\Delta \Delta C T}$ method [18]. The genes of interest were iNOS, COX-2, and IL-1 $\beta$. $\beta$-actin was used as the reference constitutive gene. The primer sequences obtained from Invitrogen ${ }^{\mathrm{TM}}$ used in this experiment were as follows: Homo sapiens nitric oxide synthase 2 (NOS2) forward CTGCTTGAGGTGGGCGG, Homo sapiens nitric oxide synthase 2 (NOS2) reverse GTGACTCTGACTCGGGACGCC; Homo sapiens cyclooxygenase-2 (Cox-2) forward CCCTTGGGTGTCAAAGGTAA, Homo sapiens cyclooxygenase-2 (Cox-2) reverse GCCCTCGCTTATGATCTGTC; Homo sapiens interleukin 1 beta (IL1B) forward GGACAAGCTGAGGAAGATGC, Homo sapiens interleukin 1 beta (IL1B) reverse TCGTTATCCCATGTGTCGAA; Homo sapiens actin beta (ACTB) forward CGTGAAAAGATGACCCAGATCA and Homo sapiens actin beta (ACTB) reverse CACAGCCTGGATGGCTACGT.

\section{Statistical analysis}

Statistical differences between the groups were assessed using one-way ANOVA. Data were expressed as mean $\pm \operatorname{SEM}(n=3)$. Statistical significance was set at $p<0.05$.

\section{RESULTS}

\section{Cannabinoid content of hemp extracts}

After obtaining the extracts from all three cultivars, it was observed that PU cultivar produced the highest amount of extracts, followed by $\mathrm{HH}$ and MSM. The yield of $\mathrm{PU}, \mathrm{HH}$, and MSM were 26.12, 25.33 and $23.75 \%$, respectively. All extracts were then analyzed using GC/MS technique. The resulting chromatograms are displayed in Figure 1. After NIST library matching, the compounds present in the extracts were divided into three subgroups, monoterpenoids at retention time less than 18 min, sesquiterpenoids at retention time between 20 and $38 \mathrm{~min}$, and cannabinoids which appeared after $45 \mathrm{~min}$.

Cannabinoids were phytochemicals found in the highest amount in all hemp extracts, followed by sesquiterpenoids and monoterpenoids. The phytochemical composition of each cultivar has been clarified in Figures 2A to 2C. PU produced the highest amount of total cannabinoids and slightly higher amount of sesquiterpenoids compared to others. MSM was the cultivar with the highest amount of monoterpenoids. The major component of sesquiterpenoids was $\beta$ caryophyllene, whose peak was found at $t \sim 24.1$, labeled (1), and humelene at $\mathrm{t} 25.6$, which was labeled (2). The peak of a major cannabinoid, $\mathrm{CBD}$, was observed at $\mathrm{t} \sim 57.5$, labeled (5) while THC was monitored at $\mathrm{t} \sim 60.2$ and labeled (6) compared to authentic standard. Peaks were also detected in PU extracts; the first peak was 
at $t \sim 51.9$, labeled (3), which did not match with any substance in NIST database and the second one was at $\mathrm{t}$ 54.8, labeled (4), which probably was tetrahydrocannabivarin (THCV). The other detected peaks in hemp extracts were compared to MS spectra database and are displayed in Table 1.

Quantitative analysis performed on CBD showed that PU produced the highest amount of $\mathrm{THC}$, followed by MSM and $\mathrm{HH}$ (1.259, 0.341 and $0.302 \%$ of dry weight, respectively). Remarkably, the amount of THC in PU cultivar was $>1.0 \%$, which might not be allowed for use due to the restriction in the Acts [19]. $\mathrm{HH}$ produced a major amount of CBD, followed by PU and MSM (2.297, 2.256 and $1.879 \%$ of dry weight, respectively). PU produced the highest amount of cannabinoids, followed by $\mathrm{HH}$ and $\operatorname{MSM}$ (3.515, 2.599 and $2.220 \%$ of dried weight, respectively) (Figure 2D). Since CBD is important for modulating undesired effects of THC and quality control in term of CBD to THC ratio [20], we determined the ratio of CBD to $\mathrm{THC}$ in each cultivar. $\mathrm{HH}$ was found have the highest proportion of CBD to THC, followed by MSM, and PU (7.61, 5.51, and 1.79, respectively) (Figure 2E).

\section{Effect of hemp extracts on release of proinflammatory cytokines}

In this study, NO and PGE2 were the cytokines of interest as these biomolecules are associated with inflammation including synovitis [1]. It was determined that the appropriate concentrations of hemp extracts used in MTT assay before the experiments on SW982 cells and the appropriate concentrations for further studies were 1, 5, and $10 \mu \mathrm{g} / \mathrm{mL}$ (data not shown). After IL-1 $\beta$ induction, SW982 produced significant amounts of $\mathrm{NO}$ compared to the control group (Figure 3).

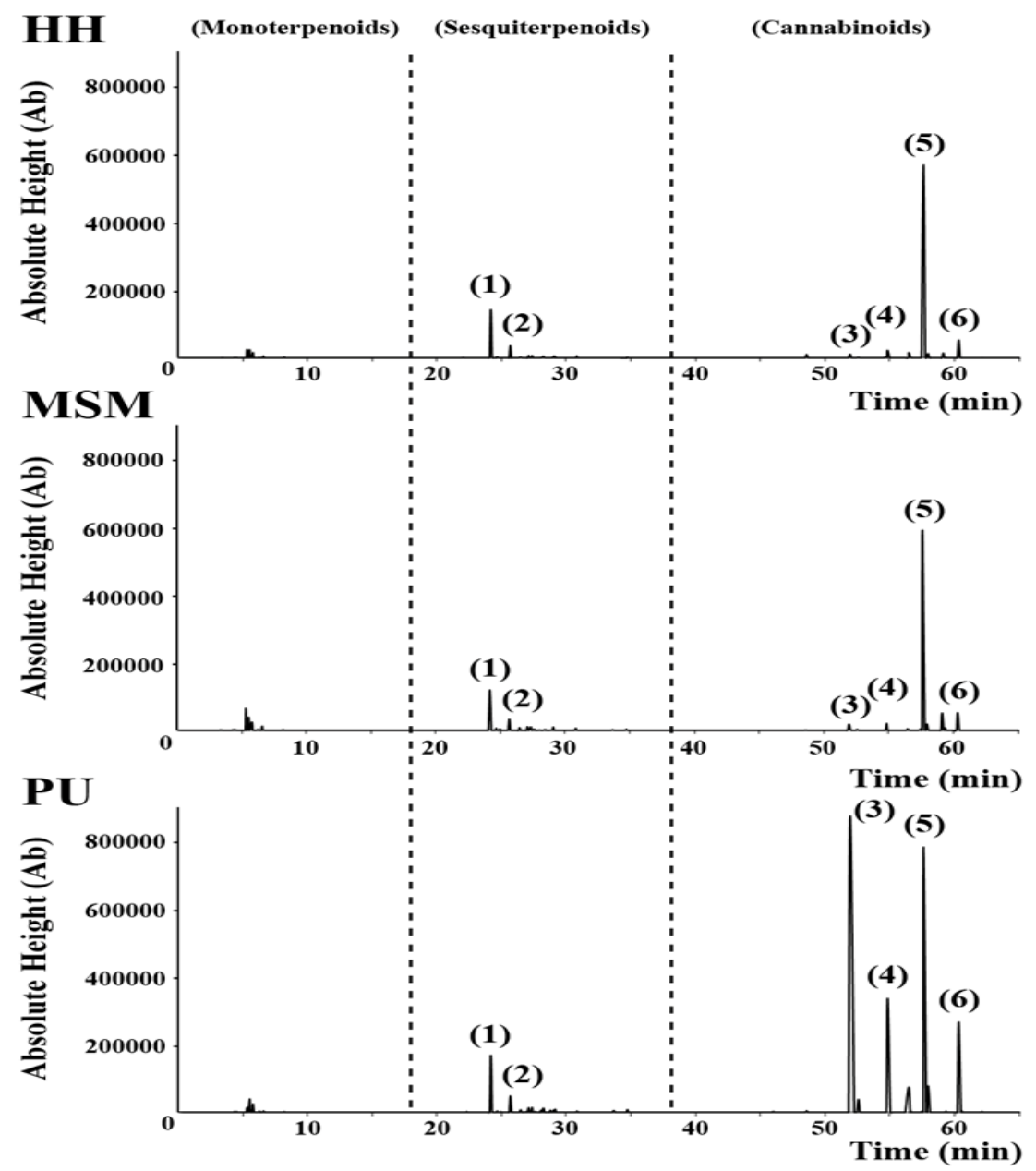

Figure 1: (A) GC chromatogram of all three hemp cultivars used in this study. The peaks detected can be divided into three subgroups using NIST database, monoterpenoids at runtime less than 18 min, sesquiterpenoids at runtime between 20 and $38 \mathrm{~min}$, and cannabinoids after $45 \mathrm{~min}$ of injection. For sesquiterpenoids, known peaks are $\beta$-caryophyllene at 24.1 min (1) and Humelene at $\mathrm{t} 25.6 \mathrm{~min}$ (2). For cannabinoids, unknown peak can be detected at $\mathrm{t} \sim 51.9 \mathrm{~min}(3)$, while the known peaks are THCV at $\mathrm{t} \sim 54.8 \mathrm{~min}(4)$, CBD at $\mathrm{t} \sim 57.5 \mathrm{~min}(5)$, and THC at $\mathrm{t} \sim 60.2 \min (6)$. 
Table 1: List of phytochemicals that matched with the NIST database. The substances are divided into subgroups related to GC chromatograms. Symbols in the table represent the appearance of substances in each hemp strain; O not detectable, + detectable, ++ detected in 2 folds of + but not more than 5 folds, and +++ detected in 5 folds of +

\begin{tabular}{|c|c|c|c|}
\hline Compound & HH & MSM & PU \\
\hline \multicolumn{4}{|l|}{ Monoterpenoids } \\
\hline a-Pinene & ++ & +++ & + \\
\hline$\beta$-Pinene & ++ & ++ & + \\
\hline Limonene & ++ & + & + \\
\hline \multicolumn{4}{|l|}{ Sesquiterpenoids } \\
\hline$\beta$-Caryophyllene & + & + & + \\
\hline$\alpha$-Bergamotene & ++ & ++ & + \\
\hline Humulene & + & + & ++ \\
\hline$\alpha$-Ylangene & + & 0 & 0 \\
\hline$\beta$-Copaene & 0 & + & + \\
\hline a-Guaiene & + & + & ++ \\
\hline$\alpha$-Farnescene & + & ++ & ++ \\
\hline a-Selinene & + & + & ++ \\
\hline$\alpha$-Cubebene & + & 0 & ++ \\
\hline Y-Eudesmol & 0 & + & + \\
\hline$\beta$-Guaiene & ++ & + & ++ \\
\hline$\alpha$-Bisabolene & + & + & + \\
\hline Caryophyllene oxide & + & + & + \\
\hline$\alpha$-Bisabolol & + & + & ++ \\
\hline \multicolumn{4}{|l|}{ Cannabinoids } \\
\hline THVC & + & + & +++ \\
\hline Cannabispiran & + & + & + \\
\hline Cannabivarin & 0 & 0 & + \\
\hline CBD & + & + & + \\
\hline $\mathrm{CBC}$ & + & + & ++ \\
\hline$\Delta^{8}-\mathrm{THC}$ & + & ++ & + \\
\hline$\Delta^{9}-\mathrm{THC}$ & + & + & ++ \\
\hline CBN & + & 0 & ++ \\
\hline
\end{tabular}

Dexamethasone at a concentration of $100 \mathrm{nM}$ could suppress the increment of NO. Interestingly, treatment with hemp extracts also contained the release of $\mathrm{NO}$ from cells in a dose dependent manner (Figure 3). NO level was clearly decreased after treatment at concentrations of 5 and $10 \mu \mathrm{g} / \mathrm{mL}$ (about 50 and $80 \%$ efficacy compared to dexamethasone, respectively). Moreover, the level of NO released after co-treatment with $10 \mu \mathrm{g} / \mathrm{mL}$ hemp extracts was almost the same as the NO level observed in the dexamethasone-treated group. PU was likely to provide the highest inhibitory effect (95 $\%$ potency of dexamethasone), although no significant differences were found among the extracts. The same tendency was detected in the determination of PGE2 (Figure 4). Remarkably, 1 $\mu \mathrm{g} / \mathrm{ml}$ of $\mathrm{HH}$ was found to reduce PGE-2 production compared to other cultivars (42\% potency of dexamethasone) while PU showed a promising result in PGE-2 reduction at $5 \mu \mathrm{g} / \mathrm{mL}$ (83\% potency of dexamethasone). There was no significant difference in PGE-2 production inhibition among the extracts at a concentration of $10 \mu \mathrm{g} / \mathrm{mL}$ (> $90 \%$ efficacy to dexamethasone).

\section{Effect of hemp extracts on iNOS, COX-2,} and IL-1 $\beta$ gene expression

In OA, IL-1 $\beta$ induced synoviocytes to produce NO and PGE2 through the iNOS and COX-2 gene upregulation. Furthermore, IL-1 $\beta$ itself can act as an autocrine, stimulating $\mathrm{IL}-1 \beta$ gene expression and increasing the IL-1 $\beta$ level [21]. Therefore, the expression of iNOS, COX-2, and IL-1 $\beta$ genes was measured by real time RTPCR. The results revealed that the extracts from all hemp cultivars affected the expression of inflammatory genes. The iNOS gene expression was increased after the cells were treated with $\mathrm{IL}-1 \beta$, but the iNOS gene expression was gradually reduced in a dose-dependent manner when co-treated with the hemp extract (Figure $5 A)$. The expressions of the COX-2 and IL- $1 \beta$ genes were also affected by the hemp extracts when they were co-treated with IL-1 $\beta$ (Figures $5 \mathrm{~B}$ and $5 \mathrm{C})$. 
$\mathbf{A}$

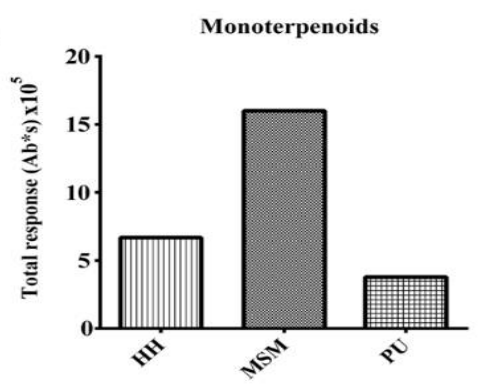

C

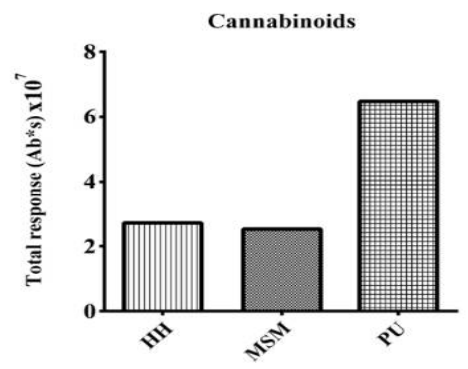

$\mathbf{E}$

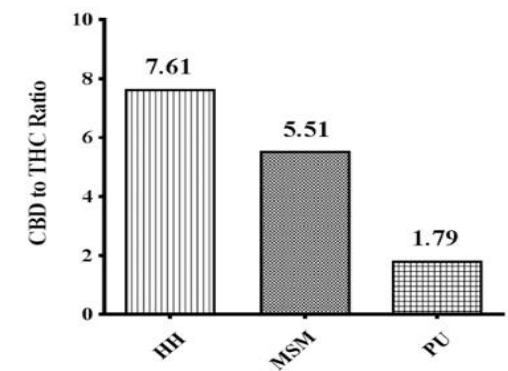

B

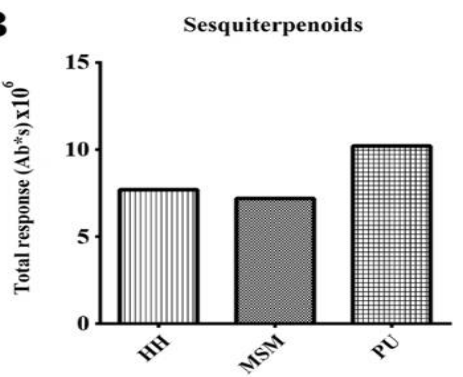

D

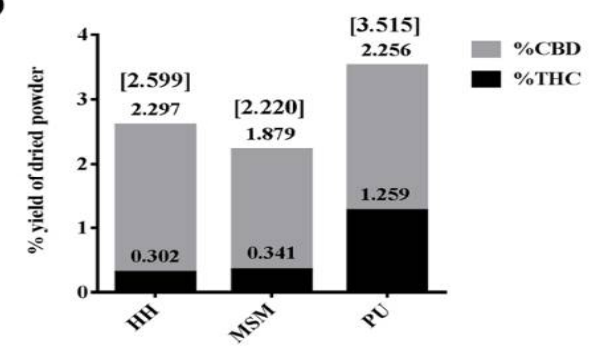

Figure 2: (A) Total monoterpenoid content detected from GC chromatogram of each hemp cultivar. (B) Total sesquiterpenoid content detected from GC chromatogram of each hemp cultivar. (C) Total cannabinoid content detected from GC chromatogram of each hemp cultivar. (D) Amount of cannabidiol (CBD) (light grey), and tetrahydrocannabinol (THC) (black) obtained from hemp extracts of each cultivar. The numbers displayed within the bracket show the total amount of CBD and THC.

(E) Ratio of CBD to THC in each cultivar

At a concentration of $5 \mu \mathrm{g} / \mathrm{mL}$, all the hemp extracts showed promising inhibitory effects (about $60 \%$ potency of dexamethasone), and at $10 \mu \mathrm{g} / \mathrm{mL}$ those effects were nearly equivalent to the effects of dexamethasone (more than $85 \%$ efficacy). Interestingly, the cultivar that contained the highest level of THC (PU) showed the greatest inhibitory effect on the expression of all indicating genes. The values corresponded to the levels of released NO. However, no significant difference in gene expression among all hemp extracts was found.

\section{DISCUSSION}

Synovitis is considered to be a major factor of $\mathrm{OA}$ pathogenesis. Although the genesis of synovitis is still unclear, the inflamed cells are found to be involved in several symptoms and clinical signs of OA [1,2]. Inflamed synoviocytes produce IL-1 $\beta$ which can orchestrate the production of NO through the induction of iNOS gene expression. Furthermore, IL-1 $\beta$ stimulates PGE2 synthesis via the upregulation of COX-2 gene expression [21]. NO, PGE2, and IL-1 $\beta$ are pivotal cytokines in the $O A$ inflammatory process and can be used as biomarkers for its diagnosis and treatment [22]. Thus, one of the key concepts in OA therapy is to regulate these proinflammatory cytokines and gene expressions in the synovium. Nowadays, researchers have considered herbal medicine, including hemp, as a substitution for various inflammatory related diseases [10]. Although hemp can be efficiently cultivated in Thailand, this plant has been categorized as a narcotic agent class 5 by the National Narcotic Drug Prohibition Act and is absolutely restricted. It might be necessary to determine the feasibility of hemp using scientific researches. Many parts of the hemp plant can be useful for various purposes; however, the leaves are usually discarded in most of the manufacturing processes regardless of cannabinoids availability [23]. 


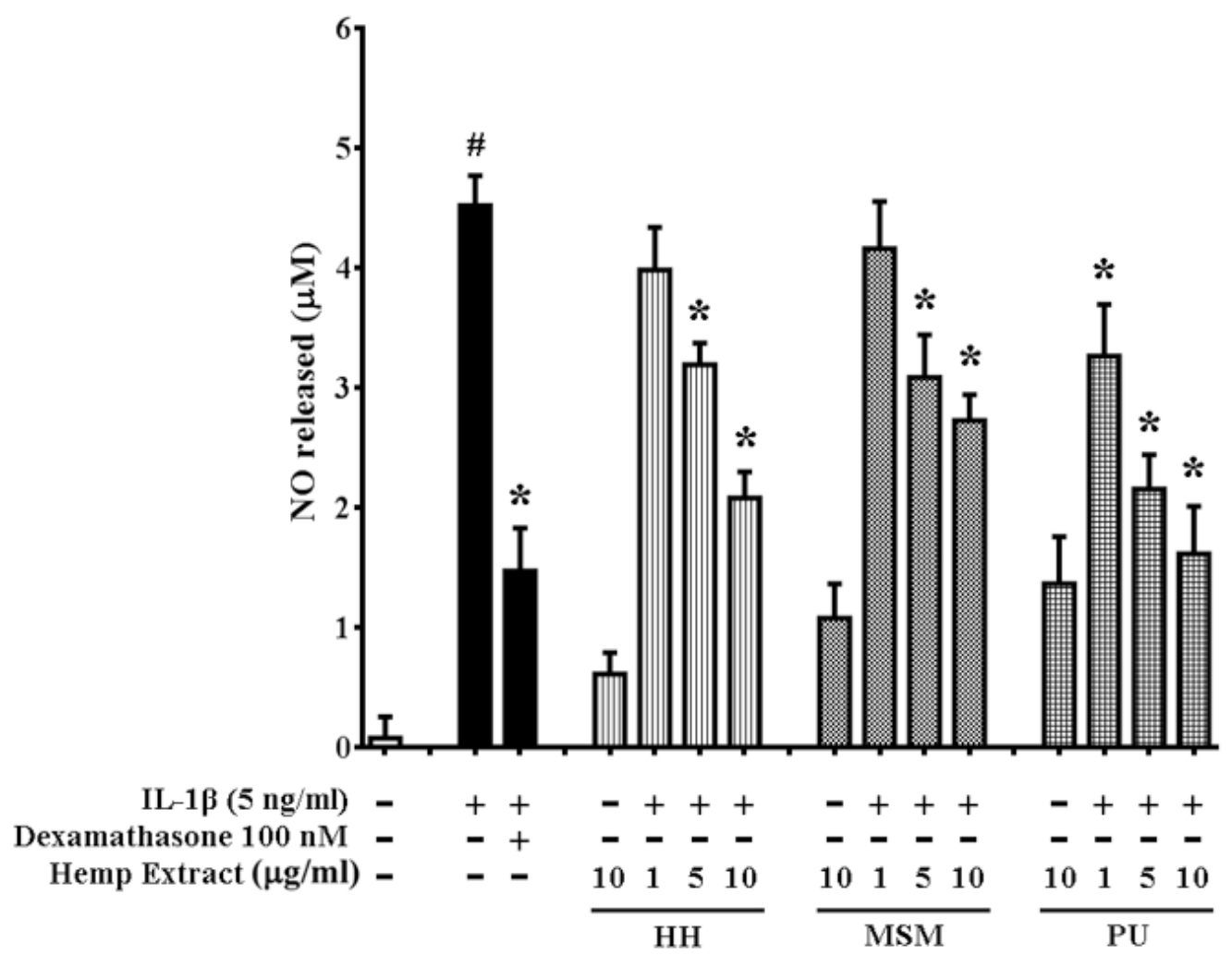

Figure 3: Effect of hemp extracts on NO synthesis from SW982 after IL-1 $\beta$ induction. Statistical significance at $p$ $<0.05$, compared to IL-1 $\beta$ treated group* and control group\#

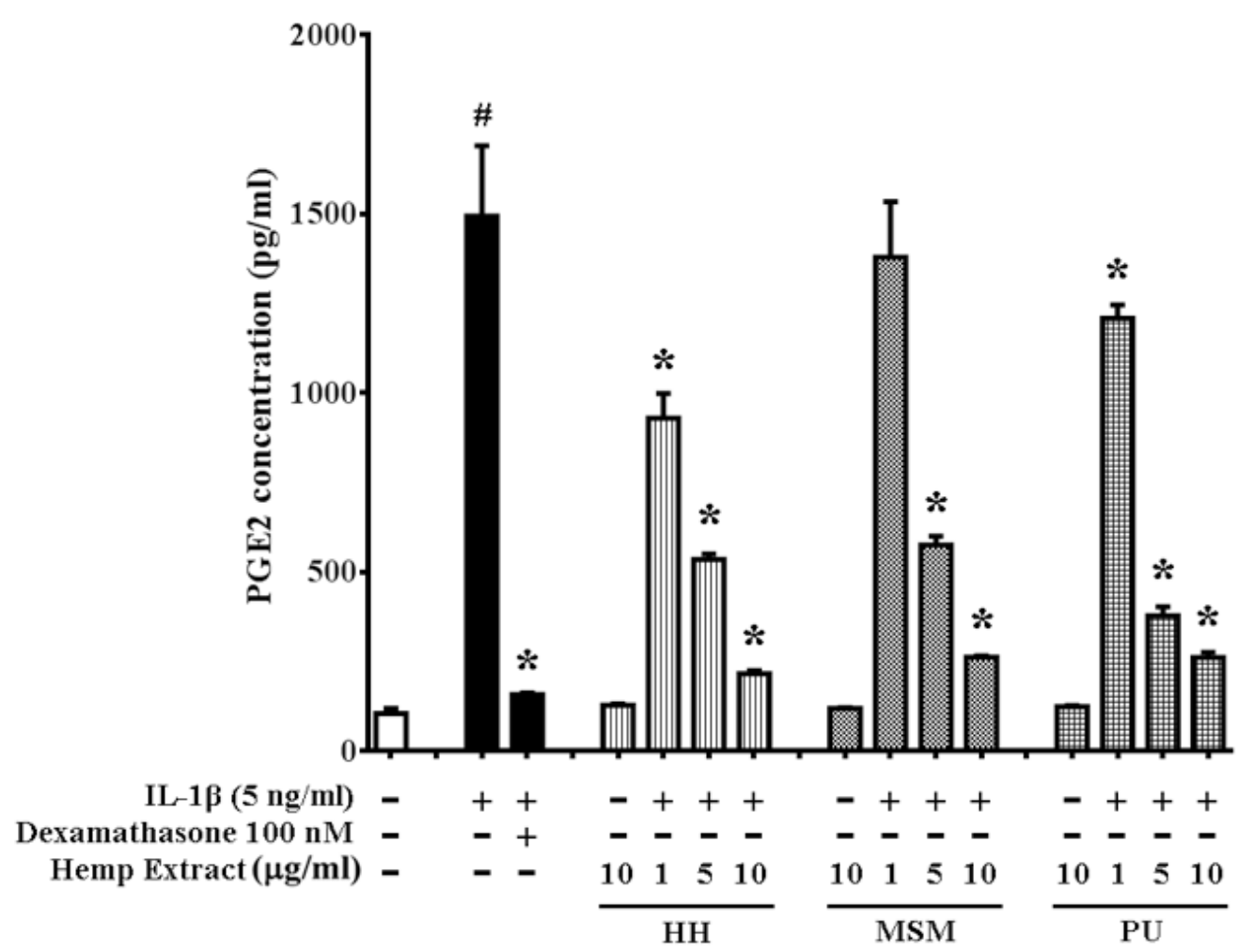

Figure 4: Effect of hemp extracts on PGE2 production from SW982 after IL-1 $\beta$ induction. Statistical significance at $p<0.05$, compared to IL-1 $\beta$ treated group* and control group\# 

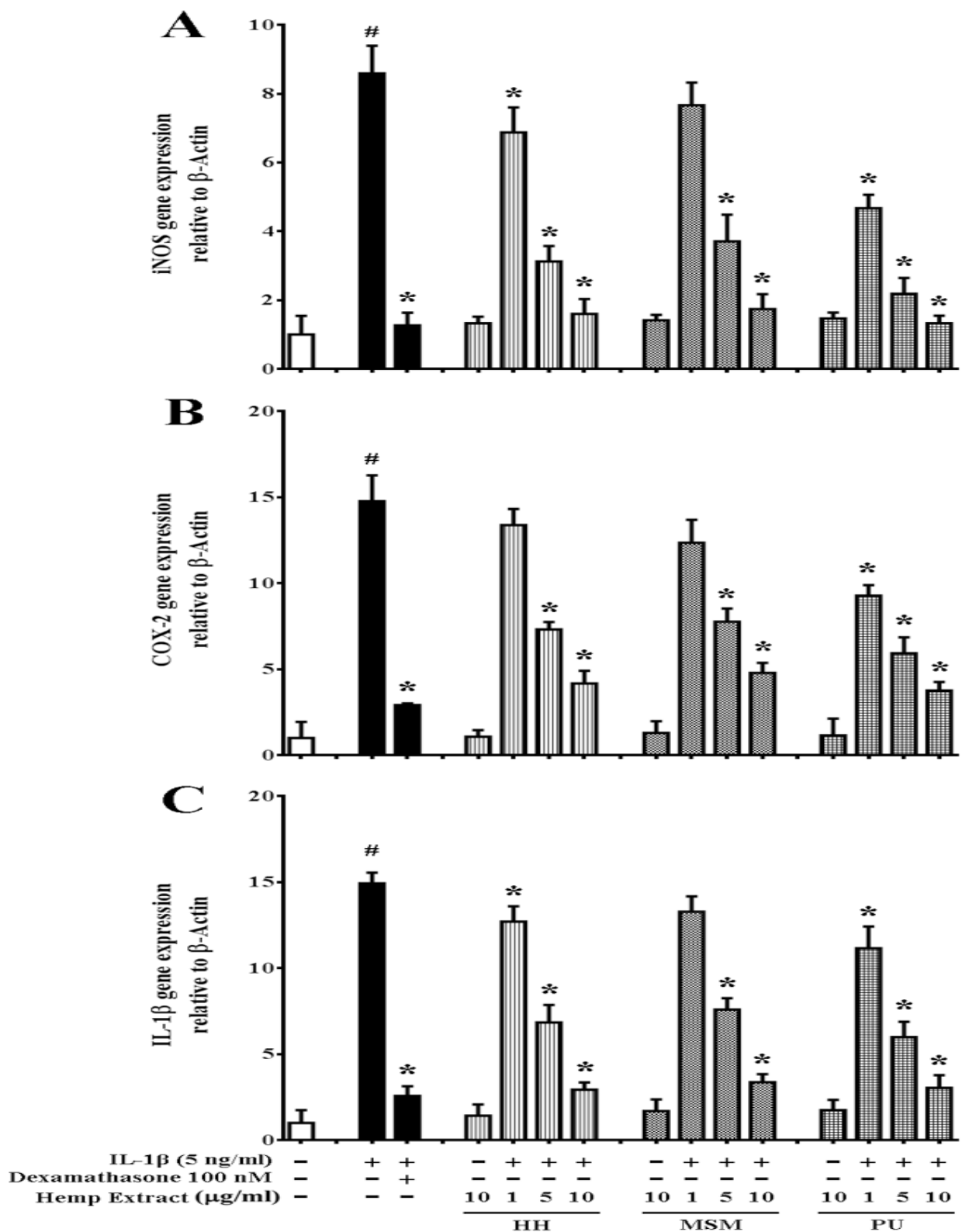

Figure 5: Effect of hemp extracts on inflammatory gene expression: (A) iNOS (B) COX-2 and (C) IL-1 13 after IL$1 \beta$ induction. The statistical significance of differences between the groups was determined using one-way ANOVA. Statistical significance at $\mathrm{P}<0.05$; * when compared to IL-1 $\beta$ treated group, \# when compared to control group

Frequently, hemp oil and hemp seed have been used in pharmacology as a neuronal analgesic and an anticonvulsant [9]. Their active ingredients are known to exhibit their effect through their receptors, CB1 and CB2, which are mostly found in the brain, neurons, and immune cells, leading to pain relief and immune balance maintenance. However, these receptors can also be found in some organs and peripheral tissues, such as endocrine glands, spleen, and heart. Moreover, CB2 receptor has been recently informed to enhance the joints of OA patients [14]. Thus, cannabinoids may provide an advantage in the treatment of OA. Moreover, THC and CBD are reported to repress the IL-1 $\beta$ and TNF- $\alpha$ production in neurons and immune cells [24]. These two cannabinoids have also been notified to inhibit not only NO production but also iNOS gene expression.

Besides, hemp consists of terpenoids, some of which were reported to be useful during inflammatory situations, including suppression of the iNOS gene upregulation [25]. These data provided the goal of this research, i.e., to determine if hemp extracts can reduce the progression of inflammation in synoviocytes. Another objective of our study was to determine whether hemp cultivation sites can affect the amount of cannabinoids in hemp leaves. For that 
reason, three hemp cultivars grown in Thailand were included in this study.

In this study, the leaves of hemp cultivars which provided a high ratio of CBD to THC were $\mathrm{HH}$, MSM, and PU. Although PU produced high amounts of cannabinoids, this cultivar also produced a high level of THC $(1.259 \%)$ which exceeds the limits specified in the Acts. Therefore, $\mathrm{HH}$ was likely to be the ideal choice as this cultivar provides high amounts of CBD and an appropriate CBD to THC ratio, although its biological activities are lower than PU. In addition, there are other cannabinoids contained in hemp plants [23]. Based on GC chromatograms, the amount of THCV was outstanding in PU cultivar, suggesting that THCV might play an important role in anti-inflammation. However, there are very few reports about the effect of THCV on arthritis. Besides, there were unidentified peaks in PU that might need to be investigated in relation to their structures and biological activities. There are also other noncannabinoid substances contained in hemp plants including terpenoids, alkaloids, flavonoids, and cardiac glycosides [26]. Interestingly, the amount of sesquiterpenoids in each of the hemp cultivar was likely to correlate with the biological responses in induced synoviocytes. This finding indicates that the effect of hemp extracts not only relied on cannabinoids, but also sesquiterpenoids. The major sesquiterpeniods found in this study were $\beta$-caryophyllene and humelene, both of which have been reported to reduce PGE2 production and iNOS and COX-2 gene expression in carrageenan injected rats [27]. Both cannabinoids and terpenoids have been reported to possess antioxidant properties which could be useful for diseases that involve reactive oxidation species (ROS), including OA.

SW932 has been identified as the optimal cell line to demonstrate synovitis under arthritis conditions and has been used in several studies [17]. Among the arthritis-related cytokines, IL-1 $\beta$ is the most well-known inflammatory cytokine which can promote self-production (autocrine), stimulate related proinflammatory genes including iNOS and COX-2, and promote synthesis of active cytokines including $\mathrm{NO}$ and PGE2 [21]. We initially determined that the concentration of $5 \mathrm{ng} / \mathrm{ml}$ of $\mathrm{IL}-1 \beta$ provided the most satisfactory condition (data not shown). A positive control used in this study was dexamethasone. The mechanism of action of dexamethasone is to inhibit the transcriptional regulators of proinflammatory genes through glucocorticoid receptor (GR) [28], which therefore suppresses the production of IL-1 $\beta$, TNF- $\alpha$ and PGE2. More so, dexamethasone reduces the expression of the iNOS gene and the NO level. From our research, dexamethasone at a concentration of $100 \mathrm{nM}$ could restore inflamed synoviocytes after being triggered with IL-1 $\beta$, as the level of IL-1 $\beta, C O X-2$ and iNOS gene expressions along with the NO and PGE2 production were restored. Interestingly, when treated with $10 \mu \mathrm{g} / \mathrm{mL}$ of hemp extracts, the level of iNOS and IL-1 $\beta$ gene expressions along with PGE2 production were nearly equivalent to that of dexamathasone-treated cells, regardless of the specific hemp cultivar. This phenomenon might involve activation of $\mathrm{CB}$ receptor, which has been found to mediate the function of GR [29].

The difference in cellular responses among cultivars could be clarified when treated at 1 and $5 \mu \mathrm{g} / \mathrm{ml}$. PU clearly inhibited the production of inflammatory mediators and expression of inflammatory genes compared to the other varieties. Although $\mathrm{HH}$ and MSM had different cannabinoid profiles, the responses of inflamed cells were virtually identical to each other. We suggest the relative amount of THC to be the dominant factor related to hemp extract activity.

After converting the CBD:THC ratio to THC:CBD, the proportions of $\mathrm{HH}, \mathrm{MSM}$, and PU were 0.131 , 0.181 , and 0.558 , respectively (data not shown). The ratio of THC to CBD of $\mathrm{HH}$ and MSM were quite similar which correlated with their cellular responses and was compatible with a previous report indicating an affinity of THC for cannabinoid receptors over CBD, especially CB2 receptor, which was overexpressed in synovitis $[12,14]$. However, this suggestion could not explain the effect of PU that contained THC about 5 times higher than other cultivars.

This study suggests that the efficacy of hemp extract on synovitis might also relate to sesquiterpenoid content. The level of sesquiterpenoids in $\mathrm{HH}$ and MSM were similar while PU contented sesquiterpenoids about 1.5 times greater than others, which corresponded with cellular responses. The level of monoterpenoids did not appear to be involved with biological responses in our study. Nonetheless, hemp extracts with a high proportion of CBD showed favorable outcomes on synovitis. HH cultivar provides promising characteristics for further investigation as it possesses high amounts of CBD, a high ratio of CBD to THC, and is able to confront the inflammation situation. This finding could establish the potency of the hemp extracts in coping with synovial inflammation. 


\section{CONCLUSION}

The findings of this study show that hemp cultivar extracts significantly reduce proinflammatory cytokines, including NO and PGE2 production, in a dose-dependent manner. In addition, the expression levels of the inflammatory genes, viz, iNOS, COX-2, and IL-1 $\beta$, declines when hemp extracts are administered to the cells. $\mathrm{HH}$ seems to be the most promising cultivar due to its phytochemical contents and biological activities. These findings indicate that hemp extracts or hemp leaf is a potential alternative treatment for inflammation-related diseases including arthritis, but further studies are required in this regard.

\section{DECLARATIONS}

\section{Acknowledgement}

The authors would like to express their gratitude to the Department of Medical Sciences, Ministry of Public Health, Thailand for research funds and Thailand Excellence Center for Tissue Engineering and Stem Cells, Faculty of Medicine, Chiang Mai University for providing facilities for carrying out the work. The authors would also like to thank Dr Robert L Lamar for suggestions and proofreading the manuscript.

\section{Conflict of Interest}

No conflict of interest associated with this work.

\section{Contribution of Authors}

The authors declare that this work was done by the authors named in this article and all liabilities pertaining to claims relating to the content of this article will be borne by them.

\section{Open Access}

This is an Open Access article that uses a funding model which does not charge readers or their institutions for access and distributed under the terms of the Creative Commons Attribution License (http://creativecommons.org/licenses/by/ 4.0) and the Budapest Open Access Initiative (http://www.budapestopenaccessinitiative.org/rea d), which permit unrestricted use, distribution, and reproduction in any medium, provided the original work is properly credited.

\section{REFERENCES}

1. Bonnet CS, Walsh DA. Osteoarthritis, angiogenesis and inflammation. Rheumatol 2005; 44: 7-16.
2. Sellam J, Berenbaum $F$. The role of synovitis in pathophysiology and clinical symptoms of osteoarthritis. Nat Rev Rheumatol 2010; 6: 625-635.

3. Scanzello CR, Goldring SR. The Role of Synovitis in Osteoarthritis pathogenesis. Bone 2012; 51 (2): 249257.

4. Plows D, Probert L, Georgopoulos S, Alexpoulou L, Kollias $G$. The role of tumour necrosis alpha (TNF) in arthritis: studies in transgenic mice. Eur $J$ Rheumatol Inflamm 1995; 2: 51-54.

5. Clancy $R$. Nitrous oxide alters chondrocyte function by disrupting cytoskeleton signaling complexes. Osteoarthr Cartil 1999; 7: 399-400.

6. Korhonen R, Lahti A, Kankaanranta H, Moilanen E. Nitric Oxide Production and Signaling in Inflammation. Curr Drug Targets 2005; 4: 471-479.

7. Rhen T, Cidlowski JA. Antiinflammatory action of glucocorticoids-new mechanisms for old drugs. $N$ Engl J Med 2005; 353: 1711-1723.

8. Russo EB. History of Cannabis and Its Preparations in Saga, Science, and Sobriquet. Chem Biodivers 2007; 4: 1614-1648.

9. Bergamaschi MM, Queiroz RHC, Crippa JAS, Zuardi AW. Safety and Side Effects of Cannabidiol, a Cannabis sativa Constituent. Curr Drug Saf 2011; 6 (4): 1-13.

10. Borgelt $L M$, Franson $K L$, Nussbaum AM, Wang GS. The Pharmacologic and Clinical Effects of Medical Cannabis. Pharmacotherapy 2013; 33 (2): 195-209.

11. Russo EB, Guy GW. A tale of two cannabinoids: the therapeutic rationale for combining tetrahydrocannabinol and cannabidiol. Med Hypotheses 2006; 6: 234-246.

12. Svíženská $I$, Dubový $P$, Suulcová $A$. Cannabinoid receptors 1 and 2 (CB1 and CB2), their distribution, ligands and functional involvement in nervous system structures - A short review. Pharmacol Biochem Behav 2008; 90: 501-511.

13. Pertwee RG. The Pharmacology of Cannabinoid Receptors and Their Ligands: an Overview. Int J Obes 2006; 30: S13-S18.

14. Fukuda S, Kohsaka H, Takayasu A, Yokoyama W, Miyabe C, Miyabe $Y$, Harigai M, Miyasaka N, Nanki T. Cannabinoid receptor 2 as a potential therapeutic target in rheumatoid arthritis. BMC Musculoskelet Disord 2014; 15: 275.

15. Callaway JC. Hempseed as a nutritional resource: An overview. Euphytica 2004; 140: 65-72.

16. Tipparat $P$, Natakankitkul $S$, Chamnivikaipong $P$, Chutiwat S. Characteristics of cannabinoids composition of Cannabis plants grown in Northern Thailand and its forensic application. Forensic Sci Int 2012; 215: 164170.

17. Chang JH, Lee KJ, Kim SK, Yoo DH, Kang TY. Validity of SW982 synovial cell line for studying the drugs against rheumatoid arthritis in fluvastatin-induced apoptosis signaling model. Indian J Med Res 2014; 139; 117-124.

18. Schefe JH, Lehmann KE, Buschmann IR, Unger $T$, Funke-Kaiser H. Quantitative real-time RT-PCR data 
analysis: current concepts and the novel "gene expression's CT difference" formula. J Mol Med 2006;

19. Holler JM, Bosy TZ, Dunkley CS, Levine B, Past MR, Jacobs A. $\Delta 9$-Tetrahydrocannabinol Content of Commercially Available Hemp Products. J Anal Toxicol 2008; 32: 428-432.

20. Niesink RJM, Laar MWv. Does Cannabidiol Protect Against Adverse Psychological Effects of THC? Front Psychiatry 2013; 4 (130): 1-8.

21. Melo-Florián A. IL-1 and its role in osteoarthritis. iMedPub Journals 2011; 1 (1:3):

22. Gitter BD, Labus JM, Lees SL, Scheetz ME. Characteristics of human synovial fibroblast activation by IL-1 beta and TNF alpha. Immunology 1989; 66 (2): 196-200.

23. Ross SA, Elsohly MA. Constituents of Cannabis sativa, a review of the natural constituents. J Pharm Sci 1995; 4 (2): 1-10.

24. Jean-Gilles L, Gran B, Constantinescu CS. Interaction between cytokines, cannabinoids and the nervous system. Immunobiology 2010; 215: 606-610.
25. Sá RdCdSe, Andrade LN, Sousa DPd. A Review on AntiInflammatory Activity of Monoterpenes. Molecules 2013; 18: 1227-1254.

26. Andre CM, Hausman J-F, Guerriero G. Cannabis sativa: The Plant of the Thousand and One Molecules. Front Plant Sci 2016; 7 (19): 1-17.

27. Vijayalaxmi A, Bakshi V, Begum N, Kowmudi V, Kumar YN, Reddy Y. Anti-Arthritic and Anti Inflammatory Activity of Beta Caryophyllene against Freund's Complete Adjuvant Induced Arthritis in Wistar Rats. J Bone Rep Recomm 2015; 1 (2): 9.

28. Coutinho AE, Chapman KE. The anti-inflammatory and immunosuppressive effects of glucocorticoids, recent developments and mechanistic insights. Mol Cell Endocrinol 2011; 335 (1): 2-13.

29. Scerif $M$, Fuzesi $T$, Thomas $J D$, Kola $B$, Grossman $A B$, Fekete $C$, Korbonits $M$. CB1 receptor mediates the effects of glucocorticoids on AMPK activity in the hypothalamus. J Endocrinol 2013; 219 (1): 79-88. 